\title{
Metadatos en revistas-e de Documentación de libre acceso
}

\author{
Por Cristòfol Rovira y Mari-Carmen Marcos
}

Resumen: Se presentan los resultados obtenidos en un estudio sobre metadatos realizado sobre 61 revistas digitales de acceso libre del ámbito de la documentación. El análisis muestra que un $59 \%$ de ellas no usa metadatos útiles para la recuperación de la información, y que sólo un $13 \%$ incluyen metadatos específicos para cada una de las páginas web de los artículos.

Palabras clave: Metadatos, Revistas digitales, Revistas de libre acceso, Información y Documentación.

Title: Metadata of open access journals in the field of information science.

Abstract: This article presents the results obtained from a metadata study of 61 open access information science journals. The analysis shows that $34 \%$ do not use metadata and only $20 \%$ have specific metadata on the web page of each article.

Keywords: Metadata, Digital Journals, Open Access Journals, Information Science.
EN ESTE ARTÍCULO presentamos los resultados de un estudio de metadatos llevado a cabo por el grupo DigiDoc ${ }^{1}$ de la Universitat Pompeu Fabra en 61 revistas digitales de libre acceso del ámbito de la información y la documentación durante los meses de diciembre de 2005 y enero de 2006. El estudio se inició planteando la siguiente hipótesis: "si los profesionales de la Documentación son quienes más interés demuestran en que las páginas web cuenten con metadatos, las revistas digitales que sirven de base a 
su disciplina usarán mayoritariamente metadatos y lo harán correctamente". Los primeros resultados están mostrando que la hipótesis no se cumple:

-El $59 \%$ de estos sitios web no usan metadatos útiles para la recuperación de la información, ya sea porque no existen, porque son sólo de tipo técnico generados automáticamente por el editor de HTML o porque los artículos están en formato PDF.

-Sólo el $13 \%$ usa metadatos específicos en las páginas web de los artículos, es decir, metadatos propios para la descripción de esa página.

Como primera conclusión podemos afirmar, pues, que hay muy pocas sedes web de revistas-e de Documentación en libre acceso que estén haciendo un uso correcto de los metadatos.

\section{Metodología}

Para realizar este trabajo hemos tenido en cuenta todas las etiquetas de metadatos que aparecen en la cabecera $(<$ head $>)$ de las páginas html. Hemos diferenciado los metadatos en html de los que están en Dublin Core y RDF, y hemos contabilizado el número de palabras y sintagmas nominales que hay en los metadatos keywords o dc.subject.

El análisis ha sido llevado a cabo con la ayuda de un software de tipo "spider" de desarrollo propio configurado para que a partir de la url de la página de inicio se analice el sitio web a través de los enlaces internos que el spider localiza y rastrea. Se han realizado tres estudios independientes:

—Un análisis de la página principal (home) de cada revista.

—Un análisis de las 50 páginas web más cercanas a la página de

\begin{tabular}{|c|c|c|c|c|c|}
$\begin{array}{c}\text { Presencia metadatos HTML y DC } \\
\text { sobre el total de sedes analizadas) }\end{array}$ & $\begin{array}{c}\text { Sin } \\
\text { meta } \\
\text { (PDF) }\end{array}$ & $\begin{array}{c}\text { Sin } \\
\text { meta } \\
\text { (HTML) }\end{array}$ & $\begin{array}{c}\text { Con } \\
\text { meta } \\
\text { HTML }\end{array}$ & $\begin{array}{c}\text { Con } \\
\text { meta } \\
\text { DC }\end{array}$ & $\begin{array}{c}\text { Con } \\
\text { meta } \\
\text { HTML y } \\
\text { DC }\end{array}$ \\
\hline $\begin{array}{l}\text { En todas las sedes web } \\
\text { Presencia de metadatos HTML y DC } \\
\text { (en sedes web con metadatos) }\end{array}$ & ------- & $3,3 \%$ & $77,0 \%$ & $1,6 \%$ & $18,0 \%$ \\
\hline mer y segundo artículo del último número & $31 \%$ & $6,6 \%$ & $42,6 \%$ & $6,6 \%$ & $13,1 \%$ \\
\hline
\end{tabular}

Tabla 1

inicio de cada sitio para conocer el uso que se hace de los metadatos de forma global.

-Un análisis de las páginas web del primer y segundo artículos del último número publicado (sólo en el caso de las revistas que ofrecen acceso a los artículos en html, no así con las que usan pdf para ofrecer los textos).

Hemos podido observar que los datos obtenidos para la totalidad del sitio web no siempre corresponden con los observados para las páginas que contienen los artículos ni con los de la página de inicio.

Las revistas digitales han sido seleccionadas de acuerdo con los siguientes criterios:

- Temática relacionada con la información y la documentación.

-Formato digital disponible en internet y con acceso libre a los artículos.

Para su localización se usaron varios directorios de publicaciones digitales, especialmente DOAJ, Temaria y New Jour.

http://www.doaj.org/

http://bidoc.ub.es/rebd/

http://gort.ucsd.edu/newjour/toc.ht $m l$

La selección final fue contrastada con el listado del artículo de Antenas Rivera $(2005)^{2}$.

Las siguientes publicaciones no pudieron ser analizadas debido a problemas técnicos para ser rastreadas con el spider: Berliner handrei- chungen zur bibliothekswissenschaft, Boletin RedIris y Journal of the Medical Library Association.

\section{Análisis de la presencia de metadatos y su tipo}

La primera pregunta que nos hicimos fue ¿usan metadatos los sitios web de estas revistas? A primera vista la respuesta es afirmativa: el $97 \%$ cuenta con algún tipo de metadato, si bien esto no significa que los metadatos se encuentren en todas las páginas; por ejemplo, el porcentaje de homepages con metadatos es del $85 \%$. Además, un $21 \%$ de las sedes Web contienen tan sólo metadatos de tipo técnico generados automáticamente por el editor de html, y un $31 \%$ presenta los artículos únicamente en formato pdf. Por tanto, el porcentaje de sedes web que tienen metadatos útiles para la recuperación de la información se reduce a un $41 \%$.

Como se muestra en el gráfico, el tipo de metadatos que usan las páginas web de los artículos es mayoritariamente $\mathrm{html}(55 \%)$, y sólo un $19,7 \%$ usa Dublin Core (DC). Concretamente el 6,6\% usa sólo DC y el $13,1 \%$ tiene DC y html al mismo tiempo. Si este mismo análisis se calcula con relación al subgrupo de sedes web con metadatos, se puede ver que la presencia de DC es mayor aunque sólo alcanza un $32 \%$ de las sedes. Por el contrario, si se analizan todas las páginas, y no tan sólo las páginas de los artículos, el porcentaje de DC es mínimo: un $1,6 \%$. 
No obstante, los datos sobre el uso de DC son mucho más altos si se calculan con relación al total de sedes web que ponen metadatos específicos en los artículos, dejando aparte aquellas revistas que no ponen ningún metadato o éstos han sido generados automáticamente por el editor de html. En este caso un $48 \%$ de las sedes web utiliza DC en los artículos. Por otro lado, se detecta una mayor presencia de DC en las páginas de los artículos que en el resto de páginas (ver tabla 1).

La codificación de los metadatos en formato RDF insertados en la página web es escasísima (no llega

\begin{tabular}{|l|c|c|l|c|c|}
\hline $\begin{array}{l}\text { Elementos de los metadatos html } \\
\text { (en todas las sedes web) }\end{array}$ & \multicolumn{3}{l|}{$\begin{array}{l}\text { Elementos de los metadatos DC } \\
\text { (en todas las sedes web) }\end{array}$} \\
\hline \multicolumn{1}{|c|}{ Elemento } & Número & Porcentaje & \multicolumn{1}{c|}{ Elemento } & Número & Porcentaje \\
\hline keywords & 208 & 26,9 & dc. subject & 156 & 20,7 \\
\hline description & 186 & 24,0 & dc.title & 144 & 19,1 \\
\hline http-equiv & 121 & 15,6 & dc.identifier & 127 & 16,8 \\
\hline author & 47 & 6,1 & dc.description & 115 & 15,2 \\
\hline generator & 47 & 6,1 & dc.creator & 79 & 10,5 \\
\hline keyword & 45 & 5,8 & dc.date & 30 & 4,0 \\
\hline
\end{tabular}

\begin{tabular}{|c|c|c|}
\hline \multicolumn{3}{|c|}{$\begin{array}{l}\text { Principales elementos de los metadatos } \\
\text { HTML } \\
\text { Análisis del primer artículo del último } \\
\text { número }\end{array}$} \\
\hline Elemento & Número & Porcentaje \\
\hline http-equiv & 36 & 28,3 \\
\hline keywords & 21 & 16,5 \\
\hline description & 19 & 15,0 \\
\hline author & 10 & 7,9 \\
\hline keyword & 8 & 6,3 \\
\hline robots & 7 & 5,5 \\
\hline generator & 5 & 3,9 \\
\hline rating & 3 & 2,4 \\
\hline
\end{tabular}

Tabla 4

\begin{tabular}{|l|c|c|}
\hline $\begin{array}{c}\text { Metadatos en } \\
\text { Keyword o } \\
\text { dc.subject }\end{array}$ & Frecuencia & \% \\
\hline world wide web & 70 & 2,3 \\
\hline internet & 69 & 2,2 \\
\hline librarianship & 62 & 2,0 \\
\hline webometrics & 52 & 1,7 \\
\hline $\begin{array}{l}\text { Web information } \\
\text { retrieval }\end{array}$ & 46 & 1,5 \\
\hline e-journal & 45 & 1,5 \\
\hline Cybermetrics & 40 & 1,3 \\
\hline journal & 36 & 1,2 \\
\hline jilt & 34 & 1,1 \\
\hline research & 33 & 1,1 \\
\hline
\end{tabular}

\begin{tabular}{|l|r|}
\hline Número de etiquetas meta por página (promedio) & \\
\hline En todas las sedes web & 4,0 \\
\hline En las sedes web que contienen metadatos & 6,1 \\
\hline En las páginas del primer artículo del último número & 7,0 \\
\hline En las sedes web con etiquetas DC & 7,3 \\
\hline En las páginas del primer artículo del último número con etiquetas DC & 10,3 \\
\hline
\end{tabular}
Tabla 2 al 7\%) y en ningún caso se ha realizado en un fichero independiente.

\section{Análisis de las etiquetas meta}

Si tomamos como muestra el grupo de sedes web que utilizan metadatos, el número promedio de etiquetas meta utilizadas es de $6 . \mathrm{Si}$ restringimos el análisis al conjunto de páginas web de los artículos, la cifra aumenta a 7 metadatos por página. Se observa que este número es mayor en las páginas que han utilizado etiquetas DC, pues en estos casos el promedio de etiquetas meta es de 7 (frente a 4 de la media global), y llega hasta 10,3 en las páginas de los artículos (ver tabla 2).

Los elementos más usados en las metaetiquetas html son keyword/keywords y description. En el caso de DC son dc.subject y dc.title. En la tabla 3 se muestra el número de elementos de cada tipo en las sedes web de las revistas, y en la tabla 4 los mismos resultados pero tomando como muestra las páginas web de los artículos.
Principales elementos de los metadatos DC Análisis del primer artículo del último número

\begin{tabular}{|l|c|c|}
\hline \multicolumn{1}{|c|}{ Elemento } & Número & Porcentaje \\
\hline dc.subject & 15 & 12,2 \\
\hline dc.identifier & 11 & 8,9 \\
\hline dc.creator & 10 & 8,1 \\
\hline dc.description & 10 & 8,1 \\
\hline dc.language & 10 & 8,1 \\
\hline dc.publisher & 10 & 8,1 \\
\hline dc.title & 10 & 8,1 \\
\hline dc.format & 9 & 7,3 \\
\hline dc.type & 9 & 7,3 \\
\hline dc.date & 6 & 4,9 \\
\hline dc.contributor & 5 & 4,1 \\
\hline \multicolumn{2}{|l}{}
\end{tabular}

\section{Palabras}

\begin{tabular}{|l|c|c|}
$\begin{array}{c}\text { en Keyword o } \\
\text { dc.subject }\end{array}$ & Frecuencia & \% \\
\hline information & 260 & 5,0 \\
\hline web & 187 & 3,6 \\
\hline journal & 129 & 2,5 \\
\hline science & 118 & 2,2 \\
\hline library & 99 & 1,9 \\
\hline electronic & 94 & 1,8 \\
\hline research & 93 & 1,8 \\
\hline internet & 77 & 1,5 \\
\hline jilt & 73 & 1,4 \\
\hline law & 73 & 1,4 \\
\hline
\end{tabular}

1,4
En las páginas web de los artículos con codificación DC se ha detectado una mayor variabilidad de elementos que en el resto de páginas: para alcanzar el $85 \%$ de las etiquetas ha sido necesario analizar hasta 11 elementos, mientras que en el resto de páginas ha sido suficiente con 6 (ver tabla 4).

\section{Análisis del contenido de los elementos keyword y dc.subject}

Se han obtenido las palabras que contienen los elementos keyword/keywords y dc.subject con el fin de identificar aque- 


\section{Años de experiencia}

Esto es lo que EBSCO ofrece.

Nuestro personal gestiona sus suscripciones a revistas electrónicas individuales o incluidas en ' paquetes de revistas, suscripciones, en papel y bases de datoś.

La Lista A-Z (A-to-Z) agrupa todos: sus recursos electrónicos en una misma lista, para que sus usuariọs localicen de manera rápida los títulos disponibles y accedan fácilmente a los contenidos. Además, la lista A-to-Z alimenta ál servidor de enlaces LinkSource ${ }^{\text {, }}$, que utilizando la norma Open'URL enlaza todos sus recursos de forma compacta e inteligente.

Experiencia, servicio, contenido, soluciones.

Hablemos hoy de sus necesidades.

CUSTOMERFOCUSEDCONTENTDRIVEN

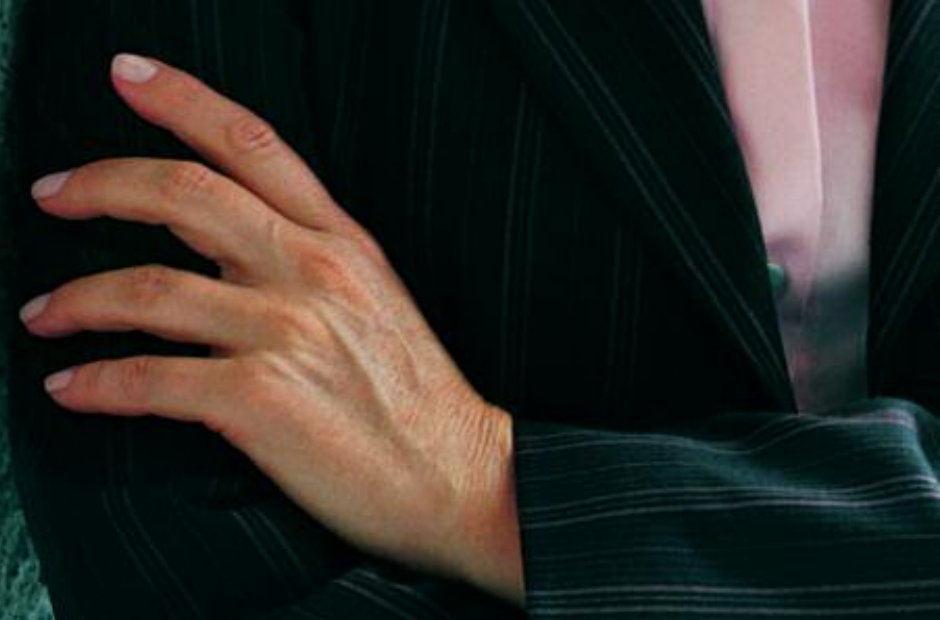
www.ebsco.com
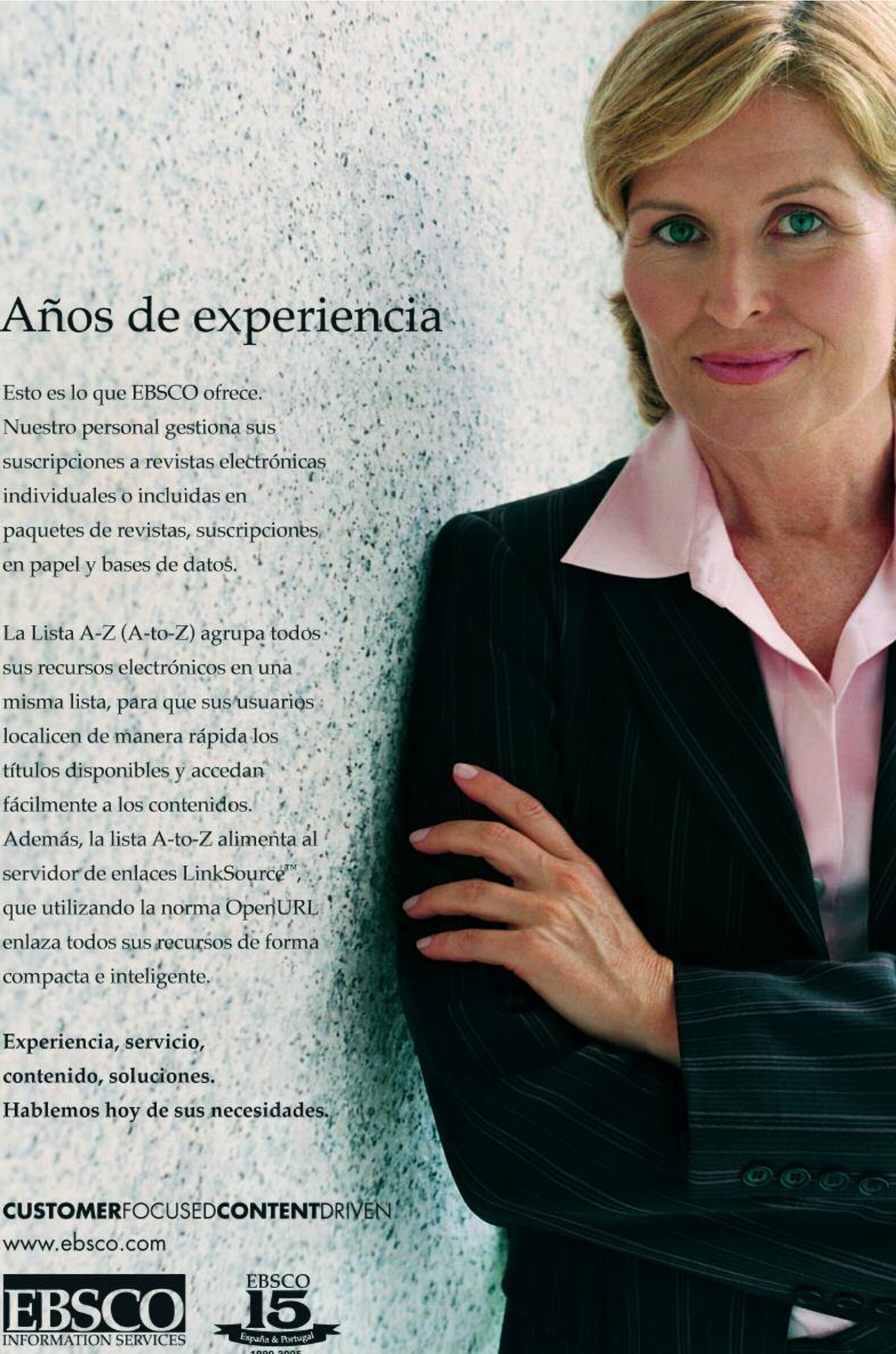


\section{Título / URL}

Acimed

http://scielo.sld.cu/scielo.php?script=sci_issuetoc\&pid=1024-

943520050004\&lng=en\&nrm=iso

Anales de Documentación

http://www.um.es/fccd/anales/

\section{Ariadne}

http://www.ariadne.ac.uk/

Arquivística.net

http://www. arquivistica.net/ojs/index.php

Australian Academic and Research Libraries

http://alia.org.au/publishing/aarl/index.html

Avisos. Noticias de la Real Biblioteca

http://www.patrimonionacional.es/realbiblioteca/

BiD: textos universitaris de Biblioteconomia i Documentació

http://www.ub.es/bid/

Biblios: Revista Electrónica de Bibliotecología y Ciencias de la

Información

http://www.bibliosperu.com/

\section{Biblioteca Universitaria}

http://dgb.unam.mx/revista/Revista\%20online/revista.html

Bibliotime Rivista elettronica per le biblioteche

http://didattica.spbo.unibo.it/bibliotime/

Biomedical Digital Libraries

http://www.bio-diglib.com/home/

Boletín de la Asociación Andaluza de Bibliotecarios

http://www.aab.es/puboletin.html

Bolletino AIB: rivista italiana di biblioteconomia e scienze

dell'informazione

http://www.aib.it/aib/boll/boll.htm

Bulletin des Bibliothèques de France

http://bbf.enssib.fr/

Bulletin of the American Society for Information Science and Technology

http://www. asis.org/Bulletin/index.html

Ciência da Informação

http://www.scielo.br/scielo.php/script_sci_serial/pid_0100-

1965/lng_en/nrm_iso

Clip boletín de la SEDIC

http://www.sedic.es/p_boletinclip.htm

Correo Bibliotecario

http://www.bcl.jcyl.es/correo/index.php

Cuadernos de documentación multimedia

http://multidoc.rediris.es/cdm/index.php

Cybermetrics

http://www.cindoc.csic.es/cybermetrics/vol9iss 1.html

D-Lib Magazine

http://www.dlib.org

\section{Digithum}

http://www.uoc.edu/digithum/

Der archivar: mitteilungsblätt für deutschen archivwesen

http://www. archive.nrw.de/archivar/

Electronic Journal of Information Systems in Developing Countries

http://new.ejisdc.org/ojs/

Electronic Journal of Know ledge Management

http://www.ejkm.com

Encontros Bibli

http://www.encontros-bibli.ufsc.br/sumario.htm

Exploit Interactive

http://www.exploit-lib.org

First Monday

http://www.firstmonday.org

High Energy Physies Libraries Webzine

http://library.cern.ch/HEPLW/

\begin{tabular}{|c|c|}
\hline$X$ & $\mathrm{X}$ \\
\hline & $\mathrm{X}$ \\
\hline \multirow[t]{2}{*}{$\mathrm{X}$} & \\
\hline & $\mathrm{X}$ \\
\hline$X$ & \\
\hline$X$ & \\
\hline \multirow[t]{2}{*}{$\mathrm{X}$} & \\
\hline & $\mathrm{X}$ \\
\hline$X$ & $X$ \\
\hline$X$ & \\
\hline \multirow[t]{2}{*}{$\mathrm{X}$} & $X$ \\
\hline & $\mathrm{X}$ \\
\hline$X$ & \\
\hline$X$ & $\mathrm{X}$ \\
\hline$X$ & \\
\hline$X$ & $X$ \\
\hline $\mathrm{X}$ & \\
\hline$X$ & $\mathrm{X}$ \\
\hline$X$ & $X$ \\
\hline$X$ & $\mathrm{X}$ \\
\hline \multirow[t]{4}{*}{$\mathrm{X}$} & \\
\hline & $X$ \\
\hline & $\mathrm{X}$ \\
\hline & $\mathrm{X}$ \\
\hline \multirow[t]{2}{*}{$X$} & $\mathrm{X}$ \\
\hline & $\mathrm{X}$ \\
\hline$X$ & \\
\hline$x$ & \\
\hline $\mathrm{X}$ & \\
\hline
\end{tabular}




\begin{tabular}{|c|c|c|}
\hline $\begin{array}{l}\text { Hipertext.net } \\
\text { http://www.hipertext.net/web/pag 100.htm }\end{array}$ & $X$ & \\
\hline $\begin{array}{l}\text { Human IT } \\
\text { http://www.hb.se/bhs/ith/humanit.htm }\end{array}$ & & $\mathrm{X}$ \\
\hline $\begin{array}{l}\text { IFLA Journal } \\
\text { http://www.ifla.org/V/iflaj/ }\end{array}$ & & $X$ \\
\hline $\begin{array}{l}\text { IT\&Society } \\
\text { http://www.stanford.edu/group/siqss/itandsociety/ }\end{array}$ & & $X$ \\
\hline $\begin{array}{l}\text { Information - Interaction - Intelligence } \\
\mathrm{http}: / / \mathrm{www} \cdot \mathrm{revue}-\mathrm{i} 3 \text {.org/index.html }\end{array}$ & & X \\
\hline $\begin{array}{l}\text { Information Research: an international electronic journal } \\
\text { http://informationr.net/ir/ }\end{array}$ & $X$ & \\
\hline $\begin{array}{l}\text { Information Technology and Disabilities } \\
\text { http://www.rit.edu/ easi/itd } . h t m\end{array}$ & X & \\
\hline $\begin{array}{l}\text { Informing Science } \\
\mathrm{http}: / / \text { inform.nu/ }\end{array}$ & & X \\
\hline $\begin{array}{l}\text { International Review of Information Ethics } \\
\text { http://www.i-r-i-e.net/ }\end{array}$ & & $X$ \\
\hline $\begin{array}{l}\text { Investigación bibliotecológica } \\
\mathrm{http}: / / \mathrm{www} \text {.ejournal.unam.mx/iibiblio/iib_v18-38.html }\end{array}$ & & $X$ \\
\hline $\begin{array}{l}\text { Issues in Science and Technology Librarianship } \\
\mathrm{http}: / / \mathrm{w} w \mathrm{w} \text {.istl.org/ }\end{array}$ & X & \\
\hline $\begin{array}{l}\text { Journal of Digital Information } \\
\text { http://jodi.tamu.edu/ }\end{array}$ & $\mathrm{X}$ & \\
\hline $\begin{array}{l}\text { Journal of Electronic Publishing } \\
\text { http://www.press.umich.edu/jep/ }\end{array}$ & $X$ & \\
\hline $\begin{array}{l}\text { Journal of Information, Law \& Technology } \\
\text { http://www2.warwick.ac.uk/fac/soc/law/elj/jilt/index.html }\end{array}$ & $\mathrm{X}$ & \\
\hline $\begin{array}{l}\text { Journal of Issues in Informing Science and Information Technology } \\
\text { http://iisit.org/ }\end{array}$ & & $X$ \\
\hline $\begin{array}{l}\text { Journal of Librarianship and Information Science } \\
\text { http://lis. sagepub.com/ }\end{array}$ & & $X$ \\
\hline $\begin{array}{l}\text { Journal of eLiteracy } \\
\mathrm{http}: / / w w w . j e l i t . o r g\end{array}$ & & X \\
\hline $\begin{array}{l}\text { Liber. Revista de bibliotecología } \\
\mathrm{http}: / / \mathrm{www} \text {.ambac.org.mx/publicaciones/liber1_4_2004.htm }\end{array}$ & & $X$ \\
\hline $\begin{array}{l}\text { Library Philosophy and Practice } \\
\text { http://www.webpages.uidaho.edu/ mbolin/lpp.htm }\end{array}$ & $X$ & $X$ \\
\hline $\begin{array}{l}\text { Libres: Library and Information Science Research Electronic Journal } \\
\text { http://libres.curtin.edu.au/ }\end{array}$ & $\mathrm{X}$ & \\
\hline $\begin{array}{l}\text { Pez de Plata: Bibliotecas Públicas a la Vanguardia } \\
\text { http://www.pezdeplata.net/portal/index.php }\end{array}$ & $X$ & $X$ \\
\hline $\begin{array}{l}\text { Revista ACB } \\
\text { http://www.acbsc.org.br/revista/ojs/index.php }\end{array}$ & & $X$ \\
\hline $\begin{array}{l}\text { Revue électronique suisse de science de l'information } \\
\mathrm{http}: / / \mathrm{campus} . \mathrm{hesge} . \mathrm{ch} / \mathrm{ressi} / \mathrm{welcome} \mathrm{html}\end{array}$ & X & $\mathrm{X}$ \\
\hline $\begin{array}{l}\text { Simile. Studies in Media \& Information Literacy Education } \\
\text { http://www.utpjournals. com/jour.ihtml? } 1 \mathrm{p}=\text { simile/simile.html }\end{array}$ & $X$ & \\
\hline $\begin{array}{l}\text { School Library Media Research } \\
\text { http://www. ala.org/ala/aasl/aaslpubsandjournals/slmrb/schoollibrary.htm }\end{array}$ & $X$ & \\
\hline $\begin{array}{l}\text { Simbiosis: Revista electrónica de ciencias de la información } \\
\mathrm{http}: / / \operatorname{simbiosis.uprrp.edu/index.htm}\end{array}$ & $X$ & \\
\hline $\begin{array}{l}\text { Sociedad de la Información } \\
\mathrm{http}: / / \mathrm{ww} w \text {. sociedadelainformacion.com/ }\end{array}$ & $X$ & \\
\hline $\begin{array}{l}\text { Solaris Information Communication } \\
\text { http://biblio-fr.info.unicaen.fr/bnum/jelec/Solaris/ }\end{array}$ & $X$ & \\
\hline $\begin{array}{l}\text { The Bonefolder, an e-journal for the bookbinder and book artist } \\
\mathrm{http}: / / \mathrm{w} w \mathrm{w} \text {.philobiblon.com/bonefolder/index.html }\end{array}$ & & X \\
\hline $\begin{array}{l}\text { The Electronic Journal of Academic and Special Librarianship } \\
\text { http://southernlibrarianship.icaap.org/ }\end{array}$ & $X$ & \\
\hline $\begin{array}{l}\text { The Library Quarterly } \\
\text { http://www.journals.uchicago.edu/LQ/journal/home.html }\end{array}$ & $X$ & $X$ \\
\hline $\begin{array}{l}\text { Webology } \\
\text { http://www.webology.ir }\end{array}$ & X & \\
\hline
\end{tabular}


llos conceptos más utilizados en las revistas de documentación. Primero se han extraído los metadatos completos y después palabra por palabra (eliminando las vacías). Puede verse el ranking de los 10 más frecuentes en la tabla 5. Mientas que los descriptores más frecuentes son "world wide web", "internet" y "librarianship", las palabras más usadas son "information", "web", "journal" y "science".

\section{Artículos y metadatos}

El análisis de metadatos en las páginas de los artículos se ha podido realizar en 42 revistas (69\%), pues las otras 19 ofrecen los textos en formato pdf en lugar de en html. De este conjunto se ha analizado el primer y segundo artículos publicados en el último número de cada una de ellas. En el estudio se pone de manifiesto que el 59\% de las revistas analizadas no utiliza metadatos, bien porque no han sido incluidos, bien porque los artículos están en PDF o bien porque sólo se incluyen aquellos metadatos técnicos generados automáticamente por el editor de html. En las revistas con artículos en formato html que sí incluyen metadatos sobre los contenidos se observa que en la mayoría de los casos no se trata de metadatos específicos de descripción y descriptores para esa página en concreto, sino que toman los mismos metadatos que han utilizado en el resto del sitio web. De hecho, sólo 8 re-

\section{Presencia de metadatos en artículos}

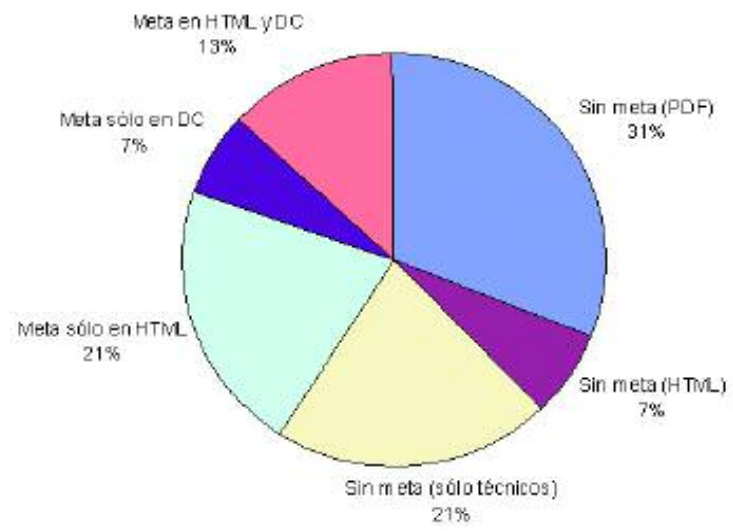

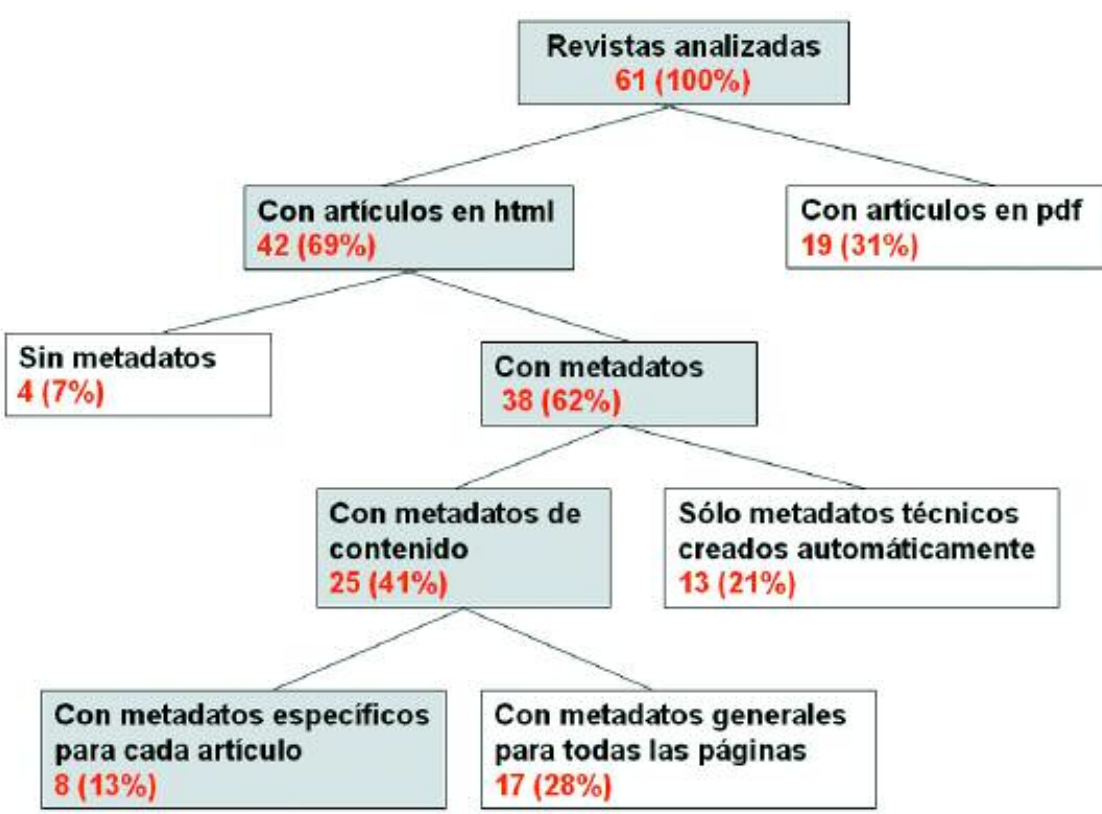

vistas (un 13\%) utilizan metadatos específicos para describir las páginas web de cada artículo publicado. Puede verse en el esquema ilustrativo cómo de la muestra total se va reduciendo el porcentaje hasta llegar a este $13 \%$ de revistas que utilizan los metadatos de forma correcta. Son las siguientes (por orden alfabético):

-Berliner handreichungen zur bi-
bliothekswissenschaft

- Cuadernos de documentación multimedia

—Exploit Interactive

-Hipertext.net

-Information Research

- Journal of Information, Law \& Technology

-Solaris Information Communication

-Webology

\section{Calidad del código HTML}

Se ha ampliado el análisis a este aspecto para detectar el número de errores de código tanto en el código html como en las hojas de estilo css. El resultado es un promedio de 40 errores de código html y de 10 errores de código de css por página, además de un promedio por página de 50 etiquetas desaconsejadas por el $W 3 C$ y un $28 \%$ de páginas sin la declaración de tipo de documento (doctype).

\section{Conclusiones}

Con esta investigación se ha puesto de relieve el poco o mal uso que las revistas del ámbito de la información y la documentación hacen de los metadatos. Consideramos necesario realizar y dar a conocer resultados de estas características, pues los profesionales de la documentación deberíamos ser los primeros en cumplir las recomendaciones que se dan desde nuestra área de conocimiento. El estudio muestra que queda camino por hacer en el uso de metadatos en las revistas digitales, más en unas que en otras, pero casi en todas se debería revisar a fondo.

Sería interesante ampliar este análisis con el estudio de otros aspectos como la usabilidad de estos sitios web, su accesibilidad o el posicionamiento web en distintos buscadores. Estamos trabajando en la mejora del spider para poder obtener más resultados. 


\section{LA NUEVA REVISTA SOBRE EL MUNDO DE LAS BIBLIOTECAS}

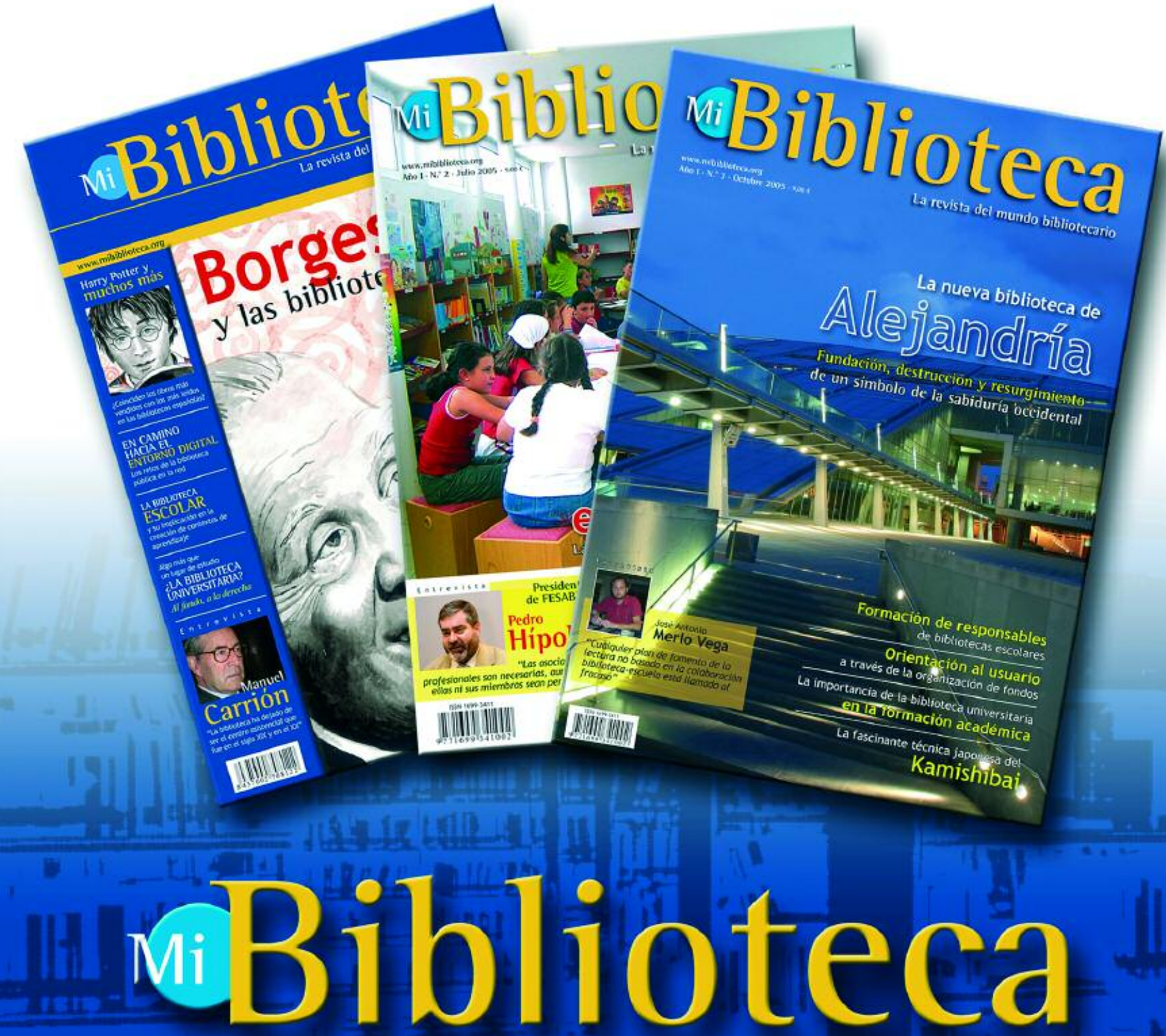

La revista del mundo bibliotecario

\section{Suscríbete.}


Esperamos que esta pincelada sobre el uso de los metadatos en las revistas digitales de documentación sirva para que entre todos mejoremos las webs de nuestra profesión. Que no se diga que "en casa del herrero, cuchillo de palo".

\section{Notas}

1. Esta investigación ha sido realizada por el Grupo DigiDoc dentro del proyecto "Web semántica y sistemas de información documental", $n^{\circ}$ HUM2004-03162/FILO del Ministerio de Educación y Ciencia (2004-2007).

2. Antenas Rivera, Javiera. "Revistas de acceso abierto en biblioteconomía y documentación". BiD: textos universitaris de bibliotecono- mia i documentació, diciembre, n. 15, 2005. Consultado en: 31-01-2006.

http://www2.ub.edu/bid/consulta_articulos.php? fichero $=15$ atena2.htm

Cristòfol Rovira y Mari-Carmen

Marcos. Universitat Pompeu Fabra. Grupo DigiDoc cristofol.rovira@upf.edu; mcarmen.marcos@upf.edu 